\title{
Volumetric Modifications of Metastatic Liver Volumes after Dramatic Responses under Chemotherapy
}

\author{
Dominique Elias, Léon Maggiori, Pascuale Misitano, Frederic Deschamps, \\ Frédéric Dumont, Michel Ducreux, Diane Goéré \\ Departments of Oncologic Surgery of Interventional Radiology and Medical Oncology, \\ Institut Gustave Roussy, Villejuif, France \\ Email: elias@igr.fr
}

Received May 3, 2011; revised October 24, 2011; accepted November 9, 2011

\begin{abstract}
Objective: To analyze the volumetric modifications of the non tumourous part of the liver when liver metastases (LM) decrease under chemotherapy. Methods: Patients were highly selected based on the following criteria: multiple bilateral large colorectal LM, response of LM attaining at least $85 \%$ under chemotherapy. The volumes and ratios of the whole liver, of the LM, and mainly of the non tumourous (normal) part of the liver, were measured on CT scan before and after chemotherapy. Results: Only ten (5\%) among 198 treated patients were eligible. Nine of them had received intraarterial chemotherapy. Metastatic involvement was initially 34\% before chemotherapy (range: $13 \%-75 \%$ ), and was 5\% (range: $1 \%$ - 25\%) after chemotherapy. The whole liver volume decreased by $41 \%$ (range: $23 \%$ - 68\%) after chemotherapy. The non metastatic liver (volume and ratio) decreased after chemotherapy in 6 patients and increased in 4 patients. The volume and ratio increased in the 4 patients whose disease initially exhibited the highest metastatic involvement $(p=0.01)$. Conclusion: The volume of the non metastatic part of the liver varied slightly under standard chemotherapy. Intra-arterial chemotherapy induces dramatic responses, but also liver injury which impairs liver regeneration. However increasing volumes were observed when initial tumour involvement was major.
\end{abstract}

Keywords: Liver Volume; Colorectal Metastases; Response under Chemotherapy

\section{Introduction}

The resectability of liver metastases (LM) has increased with progress in surgical techniques [1,2] and in chemotherapy for malignancies such as colorectal cancer [3]. Unresectability can be due to oncologic reasons (for example, a high number of LM), or to technical reasons (mainly volumetric reasons, the remaining liver is likely to be too small after an extended hepatectomy). More and more dramatic responses and sometimes complete responses of LM are observed after chemotherapy [4-6]. These responses can render, otherwise initially unresectable LM, amenable to surgery. If this hypothesis is true, the volume and ratio of the non-tumourous, normal parenchyma should progressively increase, and this may have a potential impact on the subsequent treatment strategy. To our knowledge, no study has focused on variations in the ratio of tumourous/normal liver volumes during chemotherapy.

Our objective was to show that a decreasing volume of LM is associated with an increasing volume of normal liver (non tumourous) parenchyma. We postulated that if no study has been devoted to this subject, it is because it is by no means an obvious process, but a marginal proc- ess. This is why we decided to only study "extreme" cases, i.e. highly selected patients who initially presented with numerous large bilateral LM and achieved a dramatic and unusual response after chemotherapy.

\section{Patients and Methods}

\subsection{Patients}

We retrospectively reviewed the medical files of patients with initially unresectable colorectal LM treated in our centre between January 2007 and January 2010.

Selection criteria were as follows: 1) patients with multiple bilateral large LM of colorectal origin, 2) no central portal branch compression, 3) response after chemotherapy affecting at least $85 \%$ of the volume of the metastases. An example of such a dramatic response is given in Figure 1. LM greater than $5 \mathrm{~cm}$ in diameter were present and multiple in all the patients and frequently associated with smaller LM.

\subsection{Methods}

Liver volumes were measured on CT scan performed before and after chemotherapy according to the following 

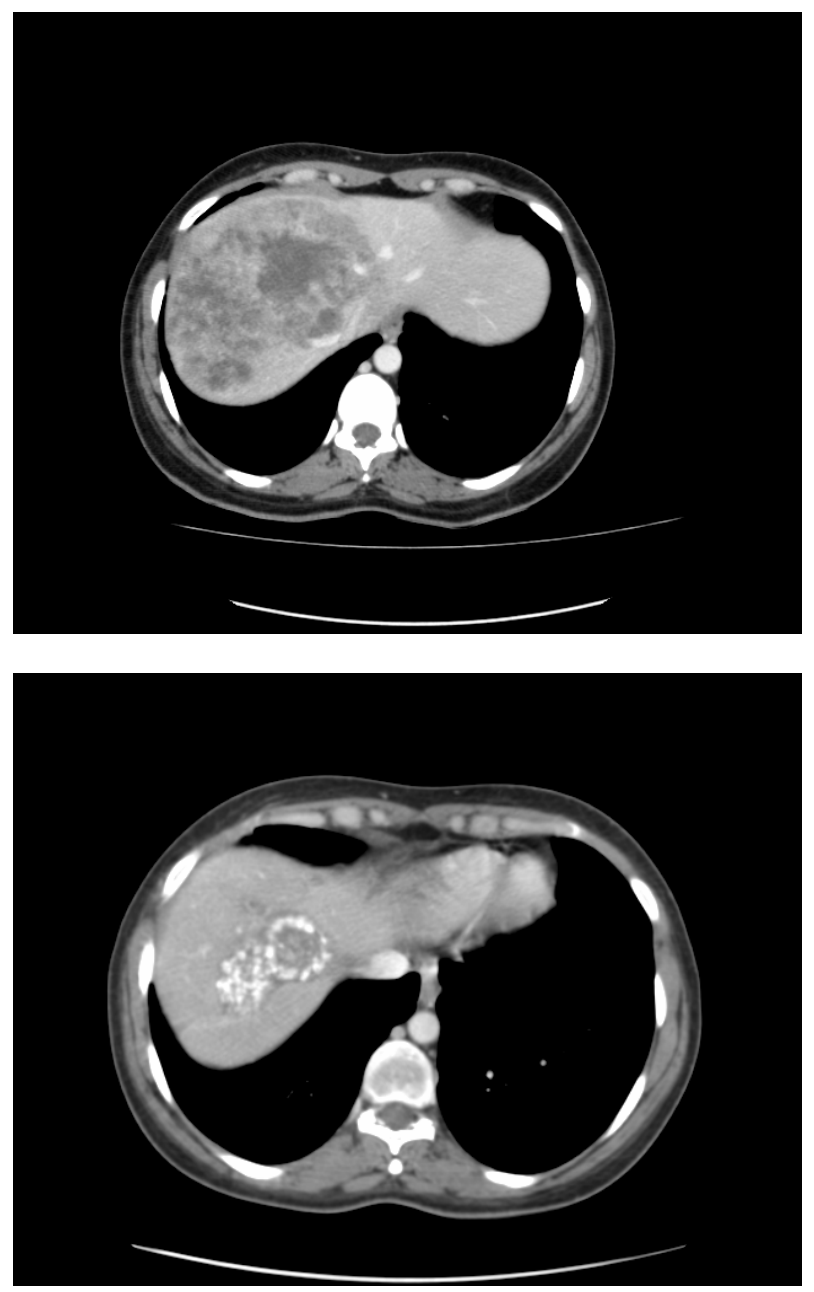

Figure 1. An example of a dramatic reduction in liver metastases under chemotherapy. method: all CT scanning was performed with a helicoidal scanner, and CT data were transferred to an independent work station for assessment. Liver and LM volumes were determined by semi-automated contouring on scans. This was performed on serial axial images: on each section, the total liver and LM were measured and the sum of the sections was calculated by integrated software. Large lesions (selection criterion) were easily measured before and after chemotherapy, but small lesions were taken into account only when they were greater than $1 \mathrm{~cm}$ of diameter. Three types of volume were assessed: the total liver volume, the volume of LM, and that of the non metastatic (normal) liver.

\subsection{Statistical Analysis}

Continuous data were compared with the Mann-Whitney $\mathrm{U}$ test. The level of statistical significance was set at $\mathrm{p}<$ 0.05 and tests were always 2-sided. The analysis was performed using the Statistical Package for the Social Sciences (SPSS, version 16.0, Chicago, IL, USA).

\section{Results}

Among 198 cases with initially unresectable LM treated during this 3-year period in our centre, only ten patients had presented with our stringent selection criteria. The decrease in the volume of LM under chemotherapy ranged from $85 \%$ to $97 \%$ (median: $93 \%$ ). The chemotherapy regimens yielding this result are reported in Table 1. It is noteworthy that nine of the patients had received intra-arterial oxaliplatin-based chemotherapy. This dramatic response under chemotherapy was obtained after first-line chemotherapy in eight of the 10 patients,

Table 1. Chemotherapy regimens.

\begin{tabular}{|c|c|c|c|c|c|}
\hline Patient & First-line & Second line & Third line & Fourth line & Total number of lines \\
\hline 1 & Oxaliplatin HAI & - & - & - & 1 \\
\hline 2 & Oxaliplatin HAI & - & - & - & 1 \\
\hline 3 & FOLFIRI + bevacizumab & Oxaliplatin HAI & - & - & 2 \\
\hline 4 & Oxaliplatin HAI & - & - & - & 1 \\
\hline 5 & Oxaliplatin HAI & - & - & - & 1 \\
\hline 6 & FOLFIRI + bevacizumab & - & - & - & 1 \\
\hline 7 & Oxaliplatin HAI & - & - & - & 1 \\
\hline 8 & FOLFIRI & FOLFOX + bevacizumab & Panitumumab & Oxaliplatin HAI & 4 \\
\hline 9 & Oxaliplatin HAI & - & - & - & 1 \\
\hline 10 & Oxaliplatin HAI & - & - & - & 1 \\
\hline
\end{tabular}

HAI Oxaliplatin: Hepatic arterial infusion (HAI) of oxaliplatin with systemic LV5FU2 and cetuximab; FOLFIRI: systemic leucovorin + fluorouracil + irinotecan; FOLFOX: systemic leucovorin + fluorouracil + oxaliplatin. 
and only once with intravenous chemotherapy (with Folfiri + bevacizumab).

Variations of the three different volumes, before and after chemotherapy, are reported in Table 2.

Metastatic involvement: Before chemotherapy, metastatic involvement ranged from $13 \%$ to $75 \%$ (median: $34 \%$ ), and after chemotherapy, it ranged from $1 \%$ to $25 \%$ (median: 5\%).

Total liver volume: Before chemotherapy, it ranged from $2260 \mathrm{ml}$ to $7053 \mathrm{ml}$ (median: $2421 \mathrm{ml}$ ). After chemotherapy, it ranged from $1308 \mathrm{ml}$ to $1984 \mathrm{ml}$ (median: $1623 \mathrm{ml}$ ), with a clear decrease in volume in all cases and median shrinkage attaining 41\% (range: 23\% - 67\%, mean: 41\%) (Table 3).

Normal liver volume (non metastatic liver): This volume represented from $25 \%$ to $87 \%$ of the total liver volume before chemotherapy (median: 66\%) (Table 2). After chemotherapy (Table 3), it increased in 4 patients (respectively by $15 \%, 18 \%, 41 \%$, and $52 \%$ ) and decreased in six patients (respectively by $9 \%, 10 \%, 19 \%$, $27 \%$, 30\%, and 30\%). No correlation was found between this increase or decrease in volumes and the following parameters: age, the chemotherapy regimen, or the duration of chemotherapy.

Table 2. Metastatic and non-metastatic (normal) liver volumes before and after chemotherapy.

\begin{tabular}{|c|c|c|c|c|c|c|}
\hline \multirow{2}{*}{ Patient } & \multicolumn{3}{|c|}{ Before chemotherapy } & \multicolumn{3}{|c|}{ After chemotherapy } \\
\hline & Total liver $^{\mathrm{a}}$ & Non metastatic (normal) liver ${ }^{\mathrm{b}}$ & Metastatic liver $^{\mathrm{b}}$ & Total liver $^{\mathrm{a}}$ & Non metastatic (normal) liver ${ }^{\mathrm{b}}$ & Metastatic liver $^{\mathrm{b}}$ \\
\hline 1 & 2346 & $1356(58 \%)$ & 990 (42\%) & 1702 & $1561(92 \%)$ & $141(8 \%)$ \\
\hline 2 & 7053 & $1770(25 \%)$ & 5284 (75\%) & 2784 & $2086(75 \%)$ & $698(25 \%)$ \\
\hline 3 & 3555 & $1240(35 \%)$ & 2315 (65\%) & 2101 & $1751(83 \%)$ & $350(17 \%)$ \\
\hline 4 & 2075 & 1759 (85\%) & $316(15 \%)$ & 1590 & $1583(98 \%)$ & $7(2 \%)$ \\
\hline 5 & 2485 & $1762(71 \%)$ & $732(29 \%)$ & 1634 & $1596(98 \%)$ & $38(2 \%)$ \\
\hline 6 & 3164 & 869 (28\%) & 2295 (72\%) & 1629 & $1322(81 \%)$ & 307 (19\%) \\
\hline 7 & 4495 & $2,714(60 \%)$ & $1781(40 \%)$ & 1459 & $1370(94 \%)$ & $90(6 \%)$ \\
\hline 8 & 2269 & $1984(87 \%)$ & $286(13 \%)$ & 1616 & 1608 (99\%) & $8(1 \%)$ \\
\hline 9 & 2336 & $1813(78 \%)$ & $523(22 \%)$ & 1308 & 1266 (97\%) & 42 (3\%) \\
\hline 10 & 2260 & 1755 (77\%) & 505 (23\%) & 1314 & 1282 (97\%) & 32 (3\%) \\
\hline Total & 2421 & 1761 (66\%) & 861 (34\%) & 1623 & 1572 (95\%) & 66 (5\%) \\
\hline
\end{tabular}

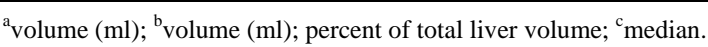

Table 3. Variations of metastatic and non-metastatic liver volumes before and after chemotherapy.

\begin{tabular}{|c|c|c|c|}
\hline Patient & Total liver $^{\mathrm{a}}$ & Non metastatic (normal) liver ${ }^{\mathrm{b}}$ & Metastatic liver ${ }^{\mathrm{b}}$ \\
\hline 1 & $-644(-27 \%)$ & +205 (+15\%) & $-849(-86 \%)$ \\
\hline 2 & $-4269(-61 \%)$ & $+316(+18 \%)$ & $-4568(-87 \%)$ \\
\hline 3 & $-1454(-41 \%)$ & +511 (+41\%) & $-1965(-85 \%)$ \\
\hline 4 & $-485(-23 \%)$ & $-176(-10 \%)$ & $-309(-98 \%)$ \\
\hline 5 & $-861(-34 \%)$ & $-166(-9 \%)$ & $-694(-95 \%)$ \\
\hline 6 & $-1535(-49 \%)$ & $+453(+52 \%)$ & $-1988(-87 \%)$ \\
\hline 7 & $-3036(-68 \%)$ & $-1344(-30 \%)$ & $-1961(-95 \%)$ \\
\hline 8 & $-653(-29 \%)$ & $-376(-1 \%)$ & $-277(-97 \%)$ \\
\hline 9 & $-1028(-44 \%)$ & $-547(-30 \%)$ & $-480(-92 \%)$ \\
\hline 10 & $-946(-42 \%)$ & $-473(-27 \%)$ & $-473(-94 \%)$ \\
\hline Total $^{\mathrm{c}}$ & $-986(-41 \%)$ & $-172(-10 \%)$ & $-772(-93 \%)$ \\
\hline
\end{tabular}

avolume (ml); ${ }^{b}$ volume (ml); percent of total liver volume; ' $m e d i a n$. 
However, it is noteworthy that the four patients who initially had the lowest ratio of normal (non metastatic) liver [25\%, 28\%, 35\%, 58\%] were those who exhibited an increasing normal liver volume after chemotherapy and this was statistically significant $(\mathrm{p}=0.01)$.

\section{Discussion}

Our aim at the beginning of this study was to determine whether the normal liver parenchyma was invaded (and destroyed) or compressed by LM, and whether normal liver was able to grow again and to replace the tumour after response to chemotherapy. This is why we focused exclusively on very selected patients exhibiting numerous and large LM whose volume decreased by at least 85\% under chemotherapy. However, the variations in the volume and ratio of the normal liver (non-metastatic) that we observed appear to be divergent.

Our patient selection criteria focused on patients exhibiting large and multiple LM achieving a dramatic response under chemotherapy. Theoretically these "extreme" cases were the most qualified to demonstrate regeneration of the normal liver as LM were eradicated. The consequence of this extremely severe selection is that we had very few patients at our disposal. We discovered, a posteriori, that intra-arterial chemotherapy with oxaliplatin had been administered to 9 of the ten patients selected, associated with systemic chemotherapy. Clearly, the intra-arterial route was the main reason for the efficacy of chemotherapy in these patients. The use of intra-arterial chemotherapy therefore induced a selection bias giving preference to patients with a particularly impaired liver parenchyma [7,8]. Oxaliplatin is routinely administered intra-arterially in our institution and we observed that liver function measured by indocyanine green clearance is highly impaired after this intra-arterial therapy, far more than after systemic chemotherapy. This clearance was always higher than $30 \%$ at 15 min (i.e. normal value X3) after intra-arterial oxaliplatin (data not shown). This particularly impaired liver parenchyma probably does not regenerate in a manner akin to that of its normal counterparts. Finally, we are faced with a paradox: the exclusive use of intra-arterial chemotherapy yields an 85\% reduction of LM, but it also induces tissue impairment which probably hinders hypertrophy or regeneration.

Nevertheless, a few important considerations can be deduced from our study:

First, the total volume of the liver increases when there is massive tumour involvement. Constant tumour progression in the liver results in a huge liver and death through liver failure. In such cases, tumour growth is so extensive that it asphyxiates the liver parenchyma and basically tumour tissue gradually supplants liver parenchyma. This can also be deduced indirectly because the total liver volume regressed under chemotherapy in all of our patients.

Second, the total liver volume decreases when LM decrease. The first obvious effect of efficient chemotherapy was to decrease the total liver volume in every case. In our highly selected cases the mean decrease in the total volume was $41 \%$. This was not an evident assessment, considering that this volume could be stay the same if the decreasing of LM was counterbalanced by the increasing of the liver parenchyma.

Third, the volume of the non-tumourous liver varied slightly but unpredictably. In the literature, there is no report on this topic because it is difficult to study. The only precise data available concern the use of preoperative selective portal embolization (mainly of the right branch) in order to achieve hypertrophy of a small future remaining left part of the liver $[9,10]$. Briefly, portal embolization must target at least two-thirds of the liver parenchyma in order to obtain a $10 \%$ increase in the ratio of the non-embolized liver (example: the left lobe increasing from $25 \%$ to $35 \%$ ), thereby rendering initially unresectable disease, amenable to surgery [10,11]. Right preoperative portal embolization was also shown to be ineffective and unnecessary before a standardized right hepatectomy [12]. In other words, only a major and dramatic vascular modification is able to induce a moderate variation of the volume of the liver parenchyma. In the case of progressing LM, there is no such vascular change, but only invasion or compression of the liver parenchyma by tumours. Their impact on the variation of the volume of the liver parenchyma is therefore limited.

In our present study, dramatic tumour regression resulted in a decrease in the normal (non metastatic) liver parenchyma in 6 patients and an increase in 4 patients, making it difficult to conclude. However, it must be underlined that the 4 patients with an increased normal liver tissue volume were those with the lowest ratio of normal parenchyma before treatment. This must be placed in parallel with that observed after portal embolization: hypertrophy or liver regeneration is observed only when metastatic involvement affects roughly $2 / 3$ of the liver, and also when approximately $2 / 3$ of the portal branches are embolized.

The reason for the increasing process appears to be simple: liver involvement roughly exceeding 50\% induces relative liver insufficiency due to intra-hepatic tissue and vascular hyperpressure, which disappears with shrinkage of huge LM. The reason for the decreasing process is due to the hepatic toxicity induced by the intra-arterial route used to administer chemotherapy. Local chemotherapy induces fibrosis [7] and sometimes cirrhosis [8], resulting in a lack of hypertrophy or regeneration, as seen after portal embolization in a cirrhotic setting [13]. Finally, in most patients receiving chemotherapy 
for LM, the variation in the volume of the non-tumourous liver is moderate and usually remains undetected.

For the time being, there is no major therapeutic repercussion expected from this rare phenomenon of regrowth of the normal liver parenchyma. Indeed, the shrinkage of LM caused by chemotherapy is more important for modifying the treatment strategy and rendering initially unresectable LM amenable to surgery than the re-growth phenomenon. However, the speed at which the normal liver parenchyma is regenerated is so far unknown, but it is low. Recently, hypertrophy of the liver after selective portal embolization was shown to persist over at least one year after embolization [14]. In other words, the phenomenon of liver recovery is different after hepatectomy (when it is rapid) than after atrophy of one part of the liver which occurs after selective portal embolization (when it is slow). In the future, it should be possible to wait much longer than usual after response to chemotherapy before appraising liver regeneration and deciding whether resectability is viable. New strategies including "waiting" while the patient is under light maintenance chemotherapy could be developed before declaring the LM definitively unresectable for volumetric reasons.

In conclusion, the main message of our study is that a volumetric variation in the non metastatic portion of the liver is negligible after usual standard chemotherapy. A dramatic reduction of LM is obtained only with intra-arterial chemotherapy which induces liver injuries that obstruct the regeneration of the non metastatic liver. However, an increasing volume was observed in cases with initial massive metastatic involvement.

\section{REFERENCES}

[1] G. Torzilli, A. Palmisano, F. Procopio, M. Cimino, F. Botea, M. Donadon, et al., "A New Systematic Small for Size Resection for Liver Tumors Invading the Middle Hepatic Vein at Its Caval Confluence,” Annals of Surgery, Vol. 251, 2010, pp. 33-39. doi:10.1097/SLA.0b013e3181b61db9

[2] D. Elias, D. Goéré, G. Leroux, C. Dromain, S. Leboulleux, T. H. de Baere, et al., "Combined Liver Surgery and RFA for Patients with Gastroenteropancreatic Endocrine Tumors Presenting with More than 15 Metastases to the Liver," EJSO, Vol. 35, No. 10, 2009, pp. m10921097.

[3] S. Kopetz, G. J. Chang, M. J. Overman, C. Eng, D. J. Sargent, D. W. Larson, et al., "Improved Survival in Metastatic Colorectal Cancer Is Associated with Adoption of Hepatic Resection and M Proved Chemotherapy," Journal of Clinical Oncology, Vol. 27, No. 22, 2009, pp. 3677-3683. doi:10.1200/JCO.2008.20.5278

[4] D. Elias, D. Goéré, V. Boige, N. Kohneh-Sharhi, D. Malka, G. Tomasic, et al., "Outcome of Posthepatectomy
Missing Colorectal Liver Metastases after Complete Response to Chemotherapy: Impact of Adjuvant Intra-Arterial Hepatic Oxaliplatin,” Annals of Surgical Oncology, Vol. 14, No. 11, 2007, pp. 3188-3194. doi:10.1245/s10434-007-9482-9

[5] R. C. Auer, R. R. White, N. Kemeny, L. H. Schwartz, J. Shia, L. Blumgart, et al., "Predictors of a True Complete Response among Disappearing Liver Metastases from Colorectal Cancer after Chemotherapy,” Cancer, Vol. 116, No. 6, 2010, pp. 1502-1509. doi:10.1002/cncr.24912

[6] S. K. Reddy, A. S. Barbas and B. M. Lary, "Synchronous Colorectal Liver Metastases: Is It Time to Reconsider Traditional Paradigms of Management?” Annals of Surgical Oncology, Vol. 16, No. 9, 2009, pp. 2395-2410. doi:10.1245/s10434-009-0372-1PMid:19506963

[7] R. C. Martin, M. J. Edwards and K. M. McMasters, "Morbidity of Adjuvant Hepatic Arterial Infusion Pump Chemotherapy in the Management of Colorectal Cancer Metastatic to the Liver," American Journal of Surgery, Vol. 188, No. 6, 2004, pp. 714-721.

doi:10.1016/j.amjsurg.2004.08.042

[8] C. Bognel, C. Degott, P. Rougier, D. Elias, S. Grandjoan, P. Duvillard, et al., "Etude Pathologique de la Toxicité héPatique de la Chimiothérapie Intra-artéRielle Hépatique,” Gastroenterologie Clinique Et Biologique, Vol. 13, 1989, pp. 125-131.

[9] H. Kinoshita, K. Sakai, K. Hirohashi, et al., "Preoperative Portal Vein Embolization for Hepatocellular Carcinoma," World Journal of Surgery, Vol. 10, No. 5, 1986, pp. 803808. doi:10.1007/BF01655244

[10] D. Elias, D. Goere, N. Kohneh-Sahrhi and T. De Baere, "Strategies for Resection Using Portal vein Embolization: Metastatic Liver Cancer," Seminars in Interventional Radiology, Vol. 25, No. 2, 2008, pp. 123-131. doi:10.1055/s-2008-1076680

[11] D. Elias, T. De Baere, A. Roche, S. Bonvallot and P. Lasser, "Preoperative Selective Portal Vein Embolizations Are an Effective Means of Extending the Indications of Major Hepatectomy in the Normal and Injured Liver," Hepato-Gastroenterology, Vol. 45, No. 19, 1998, pp. 170-177.

[12] O. Farges, J. Belghii, R. Kianmanesh, M. Regimbeau, R. Santoro, V. Vilgrain, et al., "Portal Vein Embolization before Right Hepatectomy: Prospective Clinical Trial," Annals of Surgery, Vol. 237, No. 2, 2003, pp. 208-217. doi:10.1097/00000658-200302000-00010

[13] S. Ogata, J. Belghiti, O. Farges, D. Varma, A. Sibert and V. Vilgrain, "Sequential Arterial and Portal Vein Embolizations before Right Hepatectomy in Patients with Cirrhosis and Hepatocellular Carcinoma,” British Journal of Surgery, Vol. 93, No. 9, 2006, pp. 1091-1098. doi:10.1002/bjs.5341

[14] D. Correa, L. Schwartz, W. R. Jarnagin, S. Tuorto, R. De Matteo, M. D’angelica, et al., "Kinetics of Liver Volume Changes in the First Year after Portal Vein Embolization," Archives of Surgery, Vol. 145, No. 4, 2010, pp. 351-354. doi:10.1001/archsurg.2010.42 